\title{
Structural organization of TFL1-like genes of cowpea (Vigna unguiculata (L.) Walp.)
}

Krylova E.*, Strygina K., Khlestkina E.

Federal Research Center N.I. Vavilov All-Russian Institute of Plant Genetic Resources, Saint-Petersburg, Russia

*e-mail: e.krylova@vir.nw.ru

Key words: determinate growth habit, TFL1, ATC, BFT, cowpea, Vigna unguiculata

Motivation and Aim. Cowpea (Vigna unguiculata (L.) Walp.) is a multipurpose globally important legume crop, the interest to which has greatly increased in recent years among breeders. Stem growth habit is one of the key features in determining plant architectonics. It is an economically important trait that interconnects with stem length, flowering duration, yield, resistance to lodging, and suitability of mechanical cultivation. Mutations in Terminal Flowerl (TFL1) gene and its homologs have been demonstrated to result in indeterminacy across genera. The group of TFL1-like genes includes TFL1, ATC, and BFT. Genome of $V$. unguiculata has been sequenced, but the molecular genetic mechanisms of stem growth habit control and the role of TFL1-like genes in them are not well understood. The aim of the present study was the identification and characterization of the structural and functional organization of TFL1-like genes in cowpea genome.

Methods and Algorithms: The search of homologues sequences of TFL1 in the genome of cowpea was conducted using BLASTN algorithm in LIS - Legume Information System (https://legumeinfo.org). The multiple alignment of nucleotide and amino acid sequences was made using MULTALIN v5.4.1 The cluster analysis using the MEGAX software was based on NJ algorithm. The promoter prediction was carried out with New PLACE. The annotation of the functional domains was made using InterPro. Modelling of 3Dstructures of the predicted amino acid sequences was made carried out using SWISSMODEL.

Results. The search of homologous sequences of the TFL1 gene of Arabidopsis thaliana was carried out in the genome of cowpea. In total, 4 sequences of TFL1-like genes were identified. All identified sequences were divided into three clades. The first clade included sequences highly homologous to the TFL1 gene of A. thaliana. The other two clades included genes homologous to genes ATC and BFT of A. thaliana. All identified protein sequences had all critical amino acid residues. Based on the results of the sequence analysis of identified TFL1-like genes, including the cis-regulatory regions, it can be assumed that these genes are maintained in a functional state.

Conclusion. Identification and analysis of genes responsible for the stem growth habit are required for the successful selection of modern varieties. In the genome of cowpea, we identified duplication of TFLI gene. The obtained results confirm the high evolutionary conservatism of genes involved in the molecular genetic control of the flowering initiation. Thus, this work expands the existing understanding of the molecular genetic mechanisms underlying the maintenance of indeterminacy in the growth of the shoot apical meristem, as well as in the control of the transition to flowering.

Acknowledgements: The present review was carried out within VIR project No. 0481-2019-0001. 